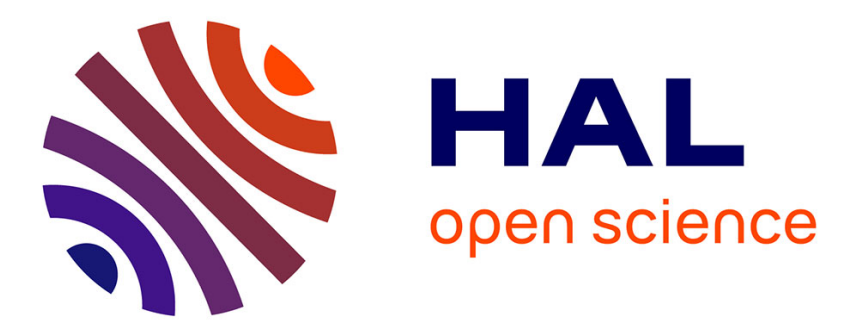

\title{
Corrélation entre commandes d'articles et citations de revues en pharmacologie
}

Pascal Bador, Chérifa Boukacem-Zeghmouri, Thierry Lafouge, Hélène Prost, Joachim Schöpfel

\section{- To cite this version:}

Pascal Bador, Chérifa Boukacem-Zeghmouri, Thierry Lafouge, Hélène Prost, Joachim Schöpfel. Corrélation entre commandes d'articles et citations de revues en pharmacologie. Therapies, 2007, 62 (4), pp.347-355. 10.2515/therapie:2007059 . hal-00588745

\section{HAL Id: hal-00588745 \\ https://hal.science/hal-00588745}

Submitted on 23 Jun 2011

HAL is a multi-disciplinary open access archive for the deposit and dissemination of scientific research documents, whether they are published or not. The documents may come from teaching and research institutions in France or abroad, or from public or private research centers.
L'archive ouverte pluridisciplinaire HAL, est destinée au dépôt et à la diffusion de documents scientifiques de niveau recherche, publiés ou non, émanant des établissements d'enseignement et de recherche français ou étrangers, des laboratoires publics ou privés.

\section{(c)(1)}

Distributed under a Creative Commons Attribution| 4.0 International License 


\section{Corrélation entre commandes d'articles et citations de revues en pharmacologie}

Pascal BADOR ${ }^{1}$; Chérifa BOUKACEM ${ }^{2}$-ZEGHMOURI ; Thierry LAFOUGE ${ }^{1}$; Hélène PROST $^{3}$; Joachim SCHÖPFEL ${ }^{3}$

1. Université de Lyon, France ; Université Lyon 1, équipe ELICO, EA 4147, Villeurbanne, F-69622, France.

2. Université Charles de Gaulle Lille, Villeneuve d'Ascq Cedex, France.

3. INIST-CNRS, Vandoeuvre Cedex, France.

\section{Correspondance :}

Pascal BADOR, Université Lyon 1, ISPB-Faculté de Pharmacie, Centre de Documentation Pharmaceutique, F-69373 Lyon Cedex 08, France.

pascal.bador@univ-lyon1.fr 


\title{
Résumé
}

Objectifs. Etudier la corrélation entre la commande d'articles à 1'INIST-CNRS et leurs citations pour 89 revues de pharmacologie et pharmacie sur la période 1992-2004. Etudier l'influence de l'arrivée de la version électronique des revues.

Méthodes. Analyse des cartes de corrélation commandes-citations des 89 revues sur 13 ans. Constitution de quatre groupes de revues.

Résultats. Les quatre groupes comportent respectivement 19, 16, 47 et 7 revues allant des revues les plus commandées et citées (les plus chères et anciennes) aux revues les moins commandées et citées. Leurs caractéristiques sont analysées en tenant compte de leur évolution sur 1992-2004. La revue Thérapie fait partie du groupe des revues très commandées mais moins citées.

Conclusion. La corrélation entre commandes et citations diminue au cours du temps. Elle est influencée par l'arrivée de la version électronique des revues.

\section{Mots clés}

Commandes d'articles, Citations, Revues, Revues électroniques, Pharmacologie

\begin{abstract}
Purpose. Investigation of the correlation between document supply by INIST-CNRS and citations of 89 pharmacology and pharmacy periodicals in 1992-2004. Investigation of the influence of electronic periodicals.

Methods. Analysis of the cards of document supply-citation correlation for the 89 periodicals during 13 years. Making of four groups of periodicals.

Results. The four groups are respectively made of 19, 16, 47 and 7 periodicals from the most supplied and cited (the more expensive and aged) to the less supplied and cited. Their characteristics are analysed taking into account their evolution during 1992-2004. The periodical Thérapie is in the group of periodicals very supplied but less cited.

Conclusion. The correlation between document supply and citations decreases during the investigated time and is influenced by the coming of electronic periodicals.
\end{abstract}

\section{Keywords}

Document supply, Citations, Periodicals, Electronic resources, Pharmacology 


\section{Introduction}

Depuis 2000, l'accès aux articles des revues scientifiques a considérablement évolué pour les chercheurs. La plupart des revues sont maintenant disponibles en version électronique et accessibles directement à partir de la base de données bibliographiques interrogée lors de la recherche documentaire. La principale limite reste cependant le nombre de revues électroniques auquel chacun peut être abonné dans le cadre de son institution de recherche, sachant que si l'accès à un article électronique n'est pas possible, la procédure classique de commande de photocopie auprès d'une bibliothèque ou d'un centre fournisseur reste bien sûr toujours d'actualité. ${ }^{[1-3]}$

Par ailleurs, les pratiques des chercheurs utilisent très largement le principe des citations des articles sur lequel est fondé le très connu facteur d'impact. Ce phénomène, outre le fait qu'il entraîne la reconnaissance d'une certaine notoriété tant pour les revues citées que pour les chercheurs eux-mêmes, constitue une méthode de recherche bibliographique à part entière qui est à l'origine de la consultation d'articles électroniques et de commandes de photocopies d'articles d'une façon plus traditionnelle. ${ }^{[4-6]}$

Dans ce contexte très évolutif, nous avons voulu étudier la relation entre les citations des revues du domaine pharmacologique et pharmaceutique comme Thérapie et les commandes des articles publiés dans ces revues auprès de l'INIST-CNRS (Institut National de l'Information Scientifique et Technique), plus grand fournisseur de documents scientifiques en France. Notre étude a donc pour objectif de confirmer l'hypothèse selon laquelle les commandes d'articles papier reflètent de moins en moins la réalité des activités de recherche dans notre discipline et donc n'est plus un indice pour la citation des revues. En d'autres termes, la consultation des revues passerait essentiellement par leur version électronique.

\section{Matériels et Méthodes}

\subsection{Echantillon}

Nous avons repris et actualisé l'échantillon de revues pharmacologiques et pharmaceutiques déjà constitué lors d'une première étude relative à l'analyse de la baisse de la fourniture de documents papier en pharmacie. ${ }^{[7]}$ Nous avons ainsi travaillé sur un échantillon de 89 revues pharmacologiques et pharmaceutiques pour lesquelles nous disposions sur la période 1992-2004 soit 13 années, de leur nombre de citations et facteur d'impact publiés dans le Journal Citation Reports (JCR) de l'Institute for Scientific Information (ISI). ${ }^{[8]}$ Nous disposions de plus du nombre de photocopies d'articles commandées et fournies par l'INIST-CNRS. Disposant de toutes les données la concernant la revue Thérapie a naturellement fait partie de notre échantillon, contrairement à une revue comme l'European Journal of Clinical Pharmacology pour laquelle un certain nombre de données de l'ISI n'étaient pas disponibles.

\subsection{Traitements}

Tout d'abord, nous avons transformé les valeurs des citations du JCR et des commandes de photocopies à l'INIST en valeurs centrées réduites. C'est-à-dire que pour 
chacune des années concernées par notre période (1992-2004), nous avons calculé la valeur moyenne des commandes et des citations des 89 revues et l'écart-type correspondant, puis nous avons centré en soustrayant la moyenne à chaque commande et citation et nous avons divisé le résultat par l'écart-type correspondant.

De cette manière, nous avons pu montrer que la nouvelle variable obtenue a pour moyenne 0 et pour écart type 1 . Cette opération est courante lorsque l'on doit comparer deux distributions numériques qui ont des échelles de valeur différentes, ce qui est le cas dans ce qui suit.

Le centre du nuage de points est 0,0 . Il y a donc des commandes et des citations négatives. Chaque revue de par la valeur de ses coordonnées, citations (axe horizontal), commandes (axe vertical) se situe dans un quadrant du plan nommé quadrant 1, quadrant 2 , quadrant 3 ou quadrant 4 suivant le sens inverse des aiguilles d'une montre.

Ce travail a été fait pour les 13 années consécutives de 1992 à 2004. Nous montrons sur la figure 1 à titre d'exemple la carte obtenue pour l'année 1999.

(figure 1)

L'étape suivante de notre travail a été de procéder à la comparaison des 13 cartes constituées. Compte tenu de la difficulté de la lecture de ces cartes, nous nous sommes intéressés à la stabilité au cours des 13 années des revues dans les quadrants. Pour ce faire, nous avons établi des indicateurs de comparaison :

- Le premier indicateur a identifié quel était le quadrant où se trouvait le plus souvent la revue scientifique (cet indicateur est l'équivalent de la médiane). Nous n'avons pas mis de seuil d'appartenance à un quadrant. Le signe des coordonnées est le seul paramètre pris en compte.

- Le second indicateur a comptabilisé le nombre de changements de quadrant, durant les 13 années. C'est-à-dire, le nombre de fois où une revue passe d'un quadrant à l'autre tout au long de notre période.

Cette méthode nous a conduit à proposer une classification des revues en quatre groupes, comme présentés ci-dessous :

- Quadrant 1 : 19 revues se trouvant le plus souvent en 13 ans en haut à droite des cartes donc les plus souvent citées et commandées.

- Quadrant 2:16 revues se trouvant le plus souvent en 13 ans en haut à gauche des cartes donc les plus souvent commandées mais moins citées.

- Quadrant $3: 47$ revues se trouvant le plus souvent en 13 ans en bas à gauche des cartes donc les moins souvent commandées et citées.

- Quadrant $4: 7$ revues se trouvant le plus souvent en 13 ans en bas à droite des cartes donc les plus souvent citées mais moins commandées. 
Dans le but de confirmer la représentativité de cette typologie, nous avons procédé à un deuxième traitement prenant en compte le nombre moyen de citations dans le JCR et le nombre moyen de commandes à l'INIST, de 1992 à 2004. Nous avons donc centré et réduit les moyennes de chaque revue des commandes et des citations sur les 13 années.

Nous avons ainsi obtenu une nouvelle carte (figure 2) qui ventile les 89 revues en quatre quadrants. Nous constatons alors que seules trois revues se trouvent dans un quadrant différent de celui du traitement précédent sur les quadrants favoris : Pharmacol Therapeut et Drug Metab Dispos passent du quadrant 2 (favori) au quadrant 1 (moyenne). J Nat Prod passe du quadrant 3 (favori) au quadrant 4 (moyenne). Ce résultat, quelque peu étonnant, montre une grande stabilité des données et confirme notre typologie.

(figure 2)

A la suite de ces deux traitements qui montrent une bonne cohérence de la répartition des revues dans les quatre groupes étudiés, nous avons analysé les caractéristiques des revues constituant les quatre groupes issus du premier traitement. La composition des quadrants favoris 1,2, 3 et 4 est présentée dans le tableau I.

(tableau I)

\section{Résultats et discussion}

3.1. Caractéristiques générales des quatre groupes

Les principales caractéristiques des quatre quadrants sont résumées dans le tableau II.

(tableau II)

Les caractéristiques détaillées des quatre quadrants sont présentées ci-dessous :

\section{- Quadrant Favori 1}

Les 19 revues de ce premier groupe sont celles qui sur 13 ans (1992-2004) sont situées, le plus grand nombre de fois, dans le quadrant 1 des revues les plus commandées et les plus citées. A titre indicatif, parmi ces revues, 12 d'entre elles (63\%) n'ont jamais quitté le quadrant 1. Ce groupe qui est constitué des revues les plus importantes pour les chercheurs du domaine pharmaceutique, est donc caractérisé par une forte stabilité sur toute la période abordée dans cet article.

Par ailleurs, les revues du quadrant 1 sont, en moyenne, les plus commandées à l'INIST (546 commandes d'articles par an) et les plus citées (11 298 citations, plus du double du nombre des citations des revues du quadrant 4). Leur prix moyen d'abonnement (2313 € selon les prix du catalogue Ulrich 2005) est très nettement 
supérieur à celui des trois autres groupes. On peut penser qu'un grand nombre de laboratoires de recherche ne souhaite pas s'abonner à ces revues et préfèrent commander leurs articles à l'INIST.

Les revues du quadrant 1 présentent par ailleurs la particularité d'être les revues les plus anciennes avec l'année 1963 comme date moyenne de création. La revue la plus ancienne étant J Pharmacol Exp Ther créée en 1909. De façon corollaire, ces revues sont connues et reconnues pour leur qualité scientifique depuis de très nombreuses années $a$ priori sans fusion ni changement de nom. Cette notoriété ancienne explique sans doute, mais en partie, le grand nombre de commandes et de citations dont elles font objet.

Les revues du Quadrant 1 sont donc les plus commandées, les plus citées, les plus chères et les plus anciennes.

- Quadrant Favori 2

Les 16 revues de ce groupe, dont fait partie la revue Thérapie, sont celles qui sur 13 ans apparaissent le plus souvent dans le quadrant 2 des revues les plus commandées mais les moins citées. Ces revues sont plus instables que celles du quadrant 1 car seules 4 d'entre elles $(25 \%)$ n'ont jamais quitté ce quadrant, 5 revues $(31 \%)$ l'ont quitté une fois, 2 revues $(13,5 \%)$ l'on quitté 2 fois, 3 revues (19\%) l'ont quitté 3 fois, 1 revue $(6 \%)$ l'a quitté 4 fois et 1 revue $(6 \%)$ l'a quitté 5 fois. Bien qu'étant plus commandées à l'INIST que la moyenne, ces revues sont moins commandées que celles du quadrant 1.

Elles sont moins citées que la moyenne de l'échantillon. Leur prix moyen

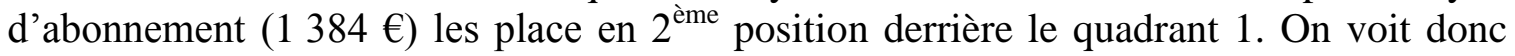
que le prix d'abonnement des revues de notre étude est plus corrélé au nombre de commandes à l'INIST qu'au nombre de citations car les revues les plus commandées (quadrants 1 et 2) sont en moyenne les plus chères.

Par ailleurs, ces revues ont une date moyenne de création de 1971 ce qui les place en $3^{\text {ème }}$ position pour leur ancienneté (revues les plus anciennes : Pharmazie et Thérapie de 1946).

\section{- Quadrant Favori 3}

Ce groupe est le plus important en nombre avec 47 revues. Elles représentent 53\% de la totalité de notre corpus. Ce sont les revues qui sont de loin les moins commandées (4 fois moins de commandes que le quadrant 1) et les moins citées (11 fois moins de citations que le quadrant 1). Les revues de ce groupe présentent une bonne stabilité sur la période abordée car 34 d'entre elles (72\%) ne l'ont jamais quitté. Le prix d'abonnement moyen est de $1050 €$ (revues les plus chères: Drug Develop Res $3900 €$, J. Labelled Compd Rad $3100 €$; revues les moins chères : Yakugaku Zasshi et Am J Pharm Educ 80 $€)$, ce sont les revues les moins chères en moyenne avec les revues du quadrant 4.

Par ailleurs, dans ce quadrant, les revues présentent une année moyenne de création de 1977, ce qui en fait le groupe des revues les plus récentes de notre corpus.

Enfin, les revues du quadrant 3 sont les moins cotées par les chercheurs. Elles doivent sans doute leur faible notoriété à leur date de création récente. 


\section{- Quadrant Favori 4}

Le quadrant 4 comporte 7 revues soit le plus petit groupe. Une seule revue n'a jamais quitté ce groupe, les autres l'ont quitté de 1 à 4 fois.

Ces revues sont moins commandées que la moyenne (moyenne de 173 commandes annuelles) mais plus citées que la moyenne.

Leur prix moyen d'abonnement est le plus bas des quatre groupes soit $972 €$.

Les quadrants 3 et 4 sont donc composés des revues les moins commandées à l'INIST et les moins chères. Leur prix étant sans doute plus abordable pour les chercheurs, ceux-ci peuvent plus facilement s'y abonner et ont donc moins besoin de commander leurs articles à l'INIST.

L'année moyenne de création des revues du quadrant 4 est 1998 (Pharmacol Rev créée en 1949); ce sont les revues en moyenne les plus anciennes après le quadrant 1.

On constate donc que les revues des quadrants 1 et 4 sont les plus anciennes et les plus citées, l'année moyenne de création a sans doute un impact sur le nombre de citations et donc sur la reconnaissance et l'utilisation des revues en terme de citations.

\subsection{Caractéristiques éditoriales}

Les caractéristiques éditoriales de notre échantillon sont basées sur les données du catologue Ulrich's ${ }^{[9]}$ et sur celles du catalogue du fonds INIST-CNRS. ${ }^{[10]}$

Les articles de l'ensemble des revues sont évalués par des experts (peer reviewed).

Le tableau III montre que la grande majorité des revues scientifiques de notre échantillon sont indexées par les sept principales bases de données bibliographiques les plus largement utilisées par les chercheurs du domaine étudié. Toutes les revues sont indexées par au moins trois de ces bases, 29 revues sont indexées par les sept bases. De plus les revues des quadrants 1 et 2 (les plus commandées) sont en pourcentage plus référencées que les revues des quadrants 3 et 4 (respectivement $91 \%$ et $94 \%$ contre $81 \%$ et $82 \%$ ).

(tableau III)

Le tableau IV montre que les revues des quadrants 1 et 2 ont une fréquence de parution et donc un nombre de numéros annuel plus important que les revues des quadrants 3 et 4 .

(tableau IV) 
Si toutes les revues du quadrant 1 sont au minimum mensuelles, les deux tiers des revues du quadrant 3 ont un maximum de dix numéros par an. Cela peut expliquer en partie le fait que les revues qui publient le plus grand nombre d'articles sont les plus citées et les plus commandées à l'INIST-CNRS.

Les revues de notre échantillon sont éditées par 35 éditeurs de 13 pays (USA $38 \%$, Royaume Uni 21\%, Allemagne 11\%, Pays Bas 9\%); 86 revues sur 89 sont anglophones.

Toutes les revues sont accessibles en texte intégral et en version électronique. En 2005, 82\% des revues mettent à disposition leurs archives au moins depuis 1999.

3.3. Analyse de l'évolution des commandes et des citations des revues sur la période 1992-2004

La figure 3 montre l'évolution du nombre moyen des commandes par quadrant et par année.

(figure 3)

Nous constatons que globalement tous les quadrants évoluent de la même façon : d'abord une croissance importante de 1992 à 1995, ensuite un pallier de 1995 à 1999, enfin une baisse de 1999 à 2004 année.

La figure 4 montre l'évolution du nombre moyen de citations par quadrant et par (figure 4)

La croissance est régulière sur la période étudiée. Le quadrant 1 se détache nettement des autres par un nombre moyen de citations plus important, mais reste dans la tendance globale à la hausse de notre échantillon.

La figure 5 montre une baisse globale régulière de la corrélation entre le nombre de commandes à l'INIST-CNRS et le nombre de citations dans le JCR de 1992 à 2004. Nous constatons en effet que le nombre moyen global de commandes augmente jusqu'en 1998 puis décroît régulièrement jusqu'en 2004. Alors que le nombre moyen global des citations augmente régulièrement sur l'ensemble de la période 1992-2004.

(figure 5) 
Une analyse plus fine par quadrants indique que les revues du quadrant 1 (revues les plus commandées et les plus citées) présentent de 1993 à 1998 une croissance faible du nombre de commandes puis une décroissance forte de 1999 à 2004 alors que leur nombre de citations augmente régulièrement et assez fortement.

L'ensemble des revues les plus commandées (quadrants 1 et 2) présente globalement une croissance assez forte du nombre de commandes de 1993 à 1998 puis une décroissance forte de 1999 à 2004 que l'on explique par la montée en charge de l'accès électronique aux revues en général et à celles de notre corpus en particulier. Parallèlement, le nombre de citations croît assez fortement de 1993 à 1998 et plus fortement encore de 1999 à 2004.

Les revues les moins commandées (quadrants 3 et 4) présentent respectivement une stabilité et une décroissance faible des commandes de 1993 à 1998 puis une décroissance assez marquée de 1999 à 2004 due également à l'arrivée des versions numériques des revues. En même temps, ces dernières présentent une faible croissance globale de leur nombre de citations de 1992 à 2004.

D'une manière générale la simple présence d'une version électronique ne devrait pas avoir d'impact sur les commandes à l'INIST car c'est l'accès (payant ou gratuit) à cette version qui détermine le besoin de commander (comme pour les abonnements papier). Si la version électronique est libre d'accès, en principe la demande devrait disparaître. Mais en principe seulement car l'INIST reçoit toujours des demandes de la part d'utilisateurs qui lui sous-traitent la recherche sur internet. Dans ce cas l'INIST fournit l'adresse du site où se trouve le document.

Les revues les plus citées (quadrants 1 et 4) présentent respectivement une quasi stabilité des commandes de 1993 à 1998 puis une forte décroissance de 1999 à 2004. Parallèlement, leur nombre de citations croit régulièrement de 1992 à 2004.

Les revues les moins citées (quadrants 2 et 3) présentent globalement une croissance modérée des commandes de 1993 à 1998 puis une décroissance importante de 1999 à 2004. Leur nombre de citations, en revanche, croît régulièrement de 1992 à 2004.

\section{Conclusion}

L'explosion de la consultation des ressources numériques est un fait avéré dans le milieu scientifique. Cependant, il présente des réalités contrastées d'un domaine à un autre. Le travail que nous avons présenté dans cet article démontre que la consultation des versions électroniques de revues traditionnelles papier peut également être contrastée à l'intérieur d'une même discipline, la pharmacologie et la pharmacie en l'occurrence.

En effet, nous avons pu voir que l'interprétation de l'éclatement du lien entre les citations et les commandes doit être faite en prenant en compte des éléments liés au fonctionnement des processus de la communication scientifique :

- Le statut de revues ancrées dans la discipline qui, de par leur ancienneté, ont historiquement contribué à la structuration d'une discipline et continuent à le faire.

- La garantie de qualité qu'elles représentent pour les chercheurs qui les considèrent comme incontournables dans leurs pratiques documentaires. 
- L’identité propre, inchangée de ces revues, malgré le phénomène de fusion des acteurs qui rythme le marché de l'édition scientifique depuis plus de 30 ans et malgré l'inflation continue du coût de ces revues.

De fait, l'analyse par quadrants que nous avons proposée dans cet article et qui souligne l'impact du numérique sur la relation commandes-citations, montre également que cet impact reste relatif sur des catégories de revues qui jouent un rôle fondateur dans le domaine. 


\section{Références}

1. Salaün JM, Lafouge T, Boukacem C. How far does demand for scientific articles reflect development in electronic publishing? An example from the Institut de l'Information Scientifique et Technique (France). Scientometrics 2000; 47(3): 561-88.

2. Bador $\mathrm{P}$, Ben Romdhane $\mathrm{M}$, Lafouge $\mathrm{T}$. European pharmaceutical journals: relationship between demand and indexation: the example of the main French document supplier. Canadian Journal of Information and Library Science 2003; 27(2): 33-55.

3. Chartron G. Les chercheurs et la documentation numérique : nouveaux services et usages. Paris : Editions du Cercle de la Librairie, 2002.

4. Echeverria M, Barredo P. Online journals : their impact on document delivery. Interlending and Document Supply 2005; 33(3): 145-9.

5. Goodier R, Dean E. Changing patterns in interlibrary loan and document supply. Interlending and Document Supply 2004; 32(4): 206-14.

6. Kidd T. Does electronic journal access affect document delivery requests? Interlending and Document Supply 2003; 31(4): 264-9.

7. Boukacem-Zeghmouri C, Bador P, Lafouge T, et al. Analysis of the downward trend in document supply in pharmacology: a case study from INIST in France (part 1). Interlending and Document Supply 2006; 34(4): 177-85.

8. ISI Web of Knowledge. http://portal.isiknowledge.com/ .

9. Ulrich's Periodicals Directory. http://www.ulrichsweb.com/ .

10. ARTICLE@INIST Le catalogue des Articles et Monographies du Fonds INIST. http://services.inist.fr/public/fre/conslt.htm . 
Tableau I. Composition des quatre quadrants favoris pour les 89 revues de notre échantillon extraites de la section «Pharmacology and Pharmacy » du JCR de l'ISI de 1992 à 2004.

\begin{tabular}{|c|c|c|}
\hline \multicolumn{3}{|c|}{ Quadrant $1: 19$ revues } \\
\hline ANTIMICROB AGENTS CH & EUR J PHARMACOL & MOL PHARMACOL \\
\hline BIOCHEM PHARMACOL & INT J PHARM & PHARMACEUT RES \\
\hline BRIT J CLIN PHARMACOL & J ANTIMICROB CHEMOTH & PHARMACOL BIOCHEM BE \\
\hline BRIT J PHARMACOL & J CARDIOVASC PHARM & PSYCHOPHARMACOLOGY \\
\hline CHEM PHARM BULL & J PHARM PHARMACOL & TOXICOL APPL PHARM \\
\hline CLIN PHARMACOL THER & J PHARM SCI & \\
\hline DRUGS & J PHARMACOL EXP THER & \\
\hline \multicolumn{3}{|c|}{ Quadrant $2: 16$ revues } \\
\hline ALIMENT PHARM THERAP & J CLIN PHARMACOL & PHARMAZIE \\
\hline ARZNEIMITTEL-FORSCH & J CLIN PSYCHOPHARM & PLANTA MED \\
\hline CLIN PHARMACOKINET & J CONTROL RELEASE & THERAPIE \\
\hline CLIN THER & J PHARMACEUT BIOMED & TOXICOLOGY \\
\hline DRUG DEV IND PHARM & PHARMACOL THERAPEUT & \\
\hline DRUG METAB DISPOS & PHARMACOTHERAPY & \\
\hline \multicolumn{3}{|c|}{ Quadrant $3: 47$ revues } \\
\hline ALCOHOL & DRUG DEVELOP RES & J NAT PROD \\
\hline AM J PHARM EDUC & DRUG EXP CLIN RES & J VET PHARMACOL THER \\
\hline ARCH PHARM & DRUG METAB REV & METHOD FIND EXP CLIN \\
\hline BIOMED CHROMATOGR & DRUG SAFETY & NEUROPSYCHHOPHARMACOL \\
\hline BIOMED PHARMACOTHER & EUR J DRUG METAB PH & PHARMACOL RES \\
\hline BIOPHARM DRUG DISPOS & FARMACO & PHARMACOLOGY \\
\hline CANCER CHEMOTH PHARM & FUNDAM CLIN PHARM & PHARMACOPSYCHIATRY \\
\hline CARDIOVASC DRUG REV & IMMUNOPHARM IMMUNOT & PHYTOTHER RES \\
\hline CARDIOVASC DRUG THER & INT CLIN PSYCHOPHARM & PROG NEURO-PSYCHOPH \\
\hline CHEMOTHERAPY & INT J CLIN PHARM TH & REGUL TOXICOL PHARM \\
\hline CHIRALITY & INVEST NEW DRUG & REV PHYSIOL BIOCH P \\
\hline CLIN EXP PHARMACOL P & J CHEMOTHERAPY & THER DRUG MONIT \\
\hline CLIN NEUROPHARMACOL & J CLIN PHARM THER & TOXICON \\
\hline CRIT REV THER DRUG & J ETHNOPHARMACOL & XENOBIOTICA \\
\hline CURR THER RES CLIN E & J INT MED RES & YAKUGAKU ZASSHI \\
\hline DRUG CHEM TOXICOL & J LABELLED COMPD RAD & \\
\hline \multicolumn{3}{|c|}{ Quadrant 4:7 revues } \\
\hline ANNU REV PHARMACOL & NEUROPHARMACOLOGY & TRENDS PHARMACOL SCI \\
\hline CAN J PHYSIOL PHARM & N-S ARCH PHARMACOL & \\
\hline J ANTIBIOT & PHARMACOL REV & \\
\hline
\end{tabular}


Tableau II. Principales caractéristiques par quadrants des 89 revues pharmacologiques et pharmaceutiques sur la période 1992-2004.

\begin{tabular}{|c|c|c|c|c|}
\hline Paramètre & Quadrant 1 & Quadrant 2 & Quadrant 3 & Quadrant 4 \\
\hline $\begin{array}{c}\text { Nombre de revues } \\
(\% \text { sur } 89)\end{array}$ & $\begin{array}{c}19 \\
(21) \\
\end{array}$ & $\begin{array}{c}16 \\
(18) \\
\end{array}$ & $\begin{array}{c}47 \\
(53) \\
\end{array}$ & $\begin{array}{c}7 \\
(8)\end{array}$ \\
\hline $\begin{array}{c}\text { Moyennes du nombre } \\
\text { de commandes } \\
\text { à l'INIST }\end{array}$ & $\begin{array}{c}546 \\
\text { Les plus } \\
\text { commandées }\end{array}$ & $\begin{array}{c}332 \\
\text { Les plus } \\
\text { commandées }\end{array}$ & $\begin{array}{c}131 \\
\text { Les moins } \\
\text { commandées }\end{array}$ & $\begin{array}{c}173 \\
\text { Les moins } \\
\text { commandées }\end{array}$ \\
\hline $\begin{array}{c}\text { Moyenne du nombre de } \\
\text { citations dans } \\
\text { le JCR de l'ISI }\end{array}$ & $\begin{array}{c}11298 \\
\text { Les plus } \\
\text { citées } \\
\end{array}$ & $\begin{array}{c}2488 \\
\text { Les moins } \\
\text { citées } \\
\end{array}$ & $\begin{array}{c}1066 \\
\text { Les moins } \\
\text { citées } \\
\end{array}$ & $\begin{array}{c}5449 \\
\text { Les plus } \\
\text { citées }\end{array}$ \\
\hline $\begin{array}{l}\text { Rang moyen dans } \\
\text { le classement } \\
\text { Impact Factor }\end{array}$ & 54 & 77 & 108 & 49 \\
\hline $\begin{array}{l}\text { Moyenne du prix } \\
\text { d'abonnement } \\
\text { (Ulrich 2005) }\end{array}$ & $2313 €$ & $1384 €$ & $1050 €$ & $972 €$ \\
\hline $\begin{array}{c}\text { Année moyenne } \\
\text { de création }\end{array}$ & 1963 & 1971 & 1977 & 1968 \\
\hline $\begin{array}{c}\text { Comparaison du nombre } \\
\text { de revues par rapport à } \\
\text { la moyenne des } \\
\text { commandes/citations } \\
(\% \text { sur } 89)\end{array}$ & $\begin{array}{c}21 \\
(24) \\
+2 \text { revues }\end{array}$ & $\begin{array}{c}14 \\
(16) \\
-2 \text { revues }\end{array}$ & $\begin{array}{c}46 \\
(52) \\
-1 \text { revue }\end{array}$ & $\begin{array}{c}8 \\
(9) \\
+1 \text { revues }\end{array}$ \\
\hline $\begin{array}{l}\text { Evolution des } \\
\text { commandes 1993-1998 } \\
\text { à l'INIST 1999-2004 } \\
\end{array}$ & $\begin{array}{l}\text { Crois. faible } \\
\text { Décr. forte }\end{array}$ & $\begin{array}{l}\text { Crois. forte } \\
\text { Décr. forte }\end{array}$ & $\begin{array}{l}\text { Crois. faible } \\
\text { Décr. faible }\end{array}$ & $\begin{array}{l}\text { Crois. faible } \\
\text { Décr. forte }\end{array}$ \\
\hline \begin{tabular}{lr}
\multicolumn{2}{|c|}{ Evolution des citations } \\
dans le & $1992-1998$ \\
JCR & $1999-2004$ \\
\end{tabular} & $\begin{array}{l}\text { Crois. forte } \\
\text { Crois. forte }\end{array}$ & $\begin{array}{l}\text { Crois. faible } \\
\text { Crois. forte }\end{array}$ & $\begin{array}{l}\text { Crois. faible } \\
\text { Crois. faible }\end{array}$ & $\begin{array}{l}\text { Crois. faible } \\
\text { Crois. faible }\end{array}$ \\
\hline
\end{tabular}


Tableau III. Indexation des 89 revues dans les bases de données bibliographiques

\begin{tabular}{lcc}
\hline Base de données bibliographique & $\begin{array}{c}\text { Nombre de } \\
\text { revues indexées }\end{array}$ & $\begin{array}{c}\text { Pourcentage de } \\
\text { revues indexées }\end{array}$ \\
\hline Chemical Abstracts & 87 & $98 \%$ \\
Current Contents & 87 & $98 \%$ \\
Embase & 87 & $98 \%$ \\
Medline & 86 & $97 \%$ \\
BIOSIS & 85 & $96 \%$ \\
PASCAL & 52 & $54 \%$ \\
International Pharmaceutical Abstracts & 48 & $54 \%$ \\
\hline
\end{tabular}


Tableau IV. Fréquence de parution des 89 revues

Nombre de numéros annuel Quadrant 1 Quadrant 2 Quadrant 3 Quadrant 4

\begin{tabular}{lcccc}
\hline Plus de 12 numéros par an & $32 \%$ & $25 \%$ & $4 \%$ & $14 \%$ \\
12 numéros par an & $68 \%$ & $50 \%$ & $30 \%$ & $57 \%$ \\
Moins de 12 numéros par an & $0 \%$ & $25 \%$ & $66 \%$ & $29 \%$ \\
\hline
\end{tabular}




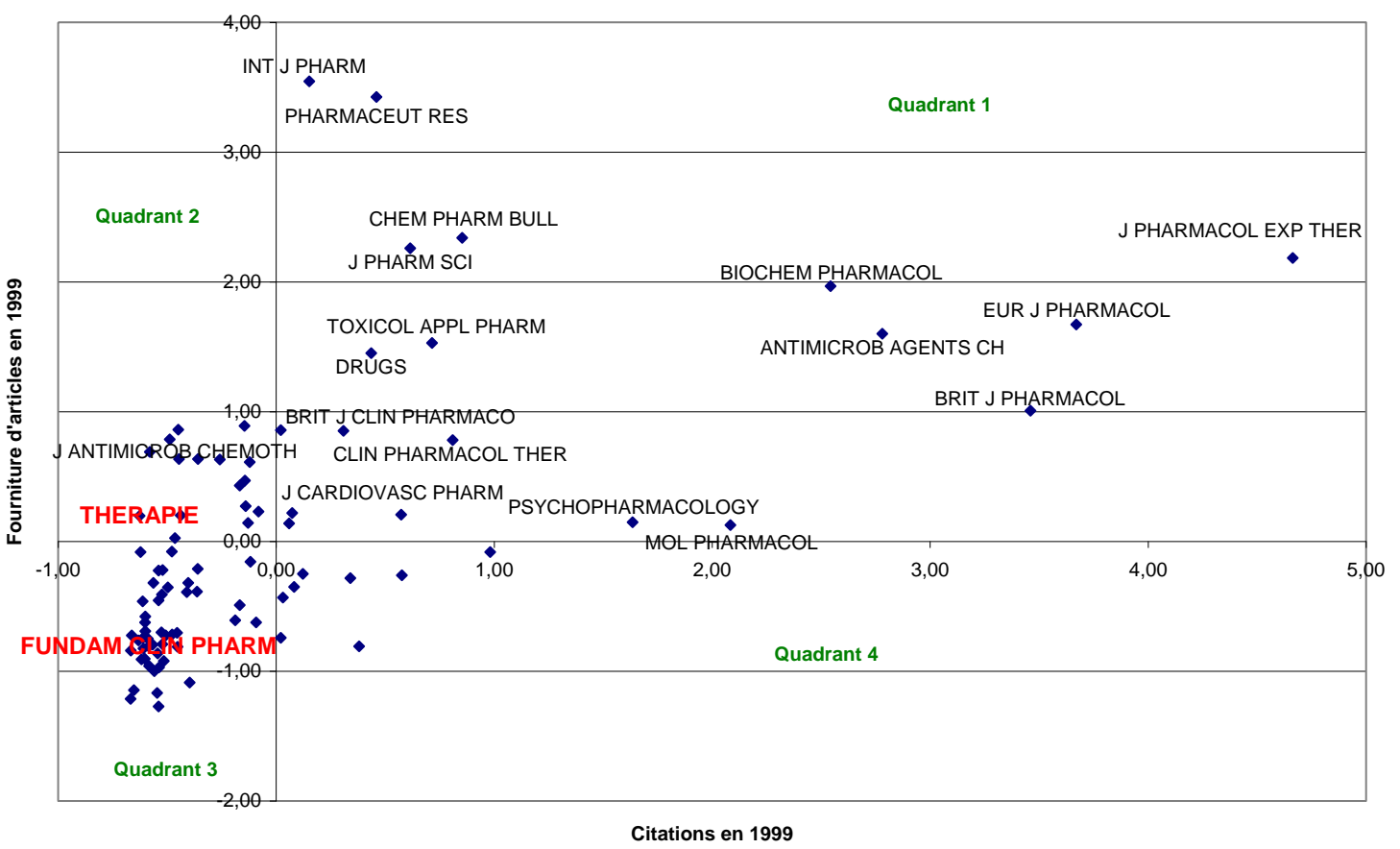

Figure 1. Données centrées réduites des commandes et des citations pour l'année 1999. 


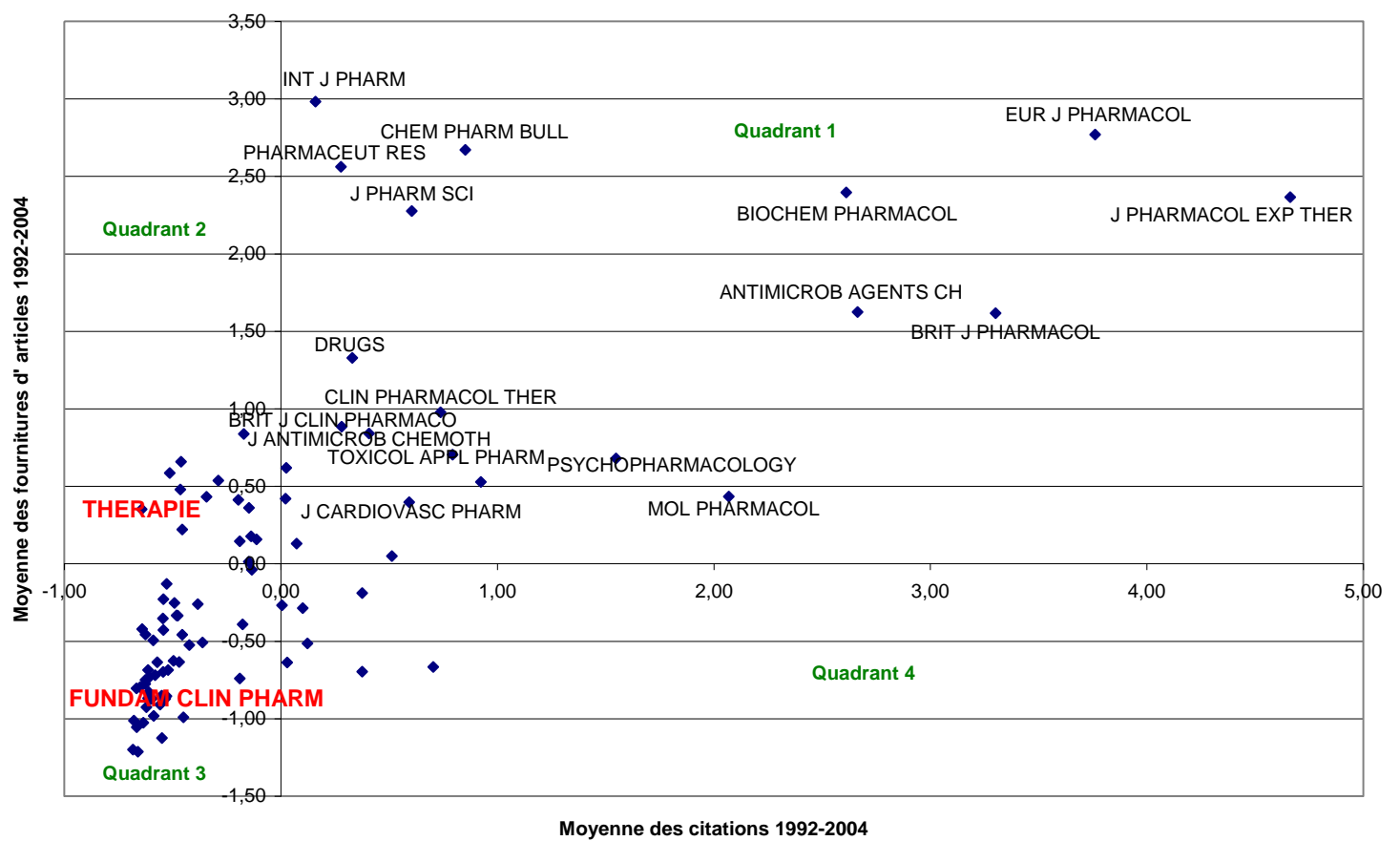

Figure 2. Données centrées réduites des moyennes des commandes et des citations sur 1992-2004. 


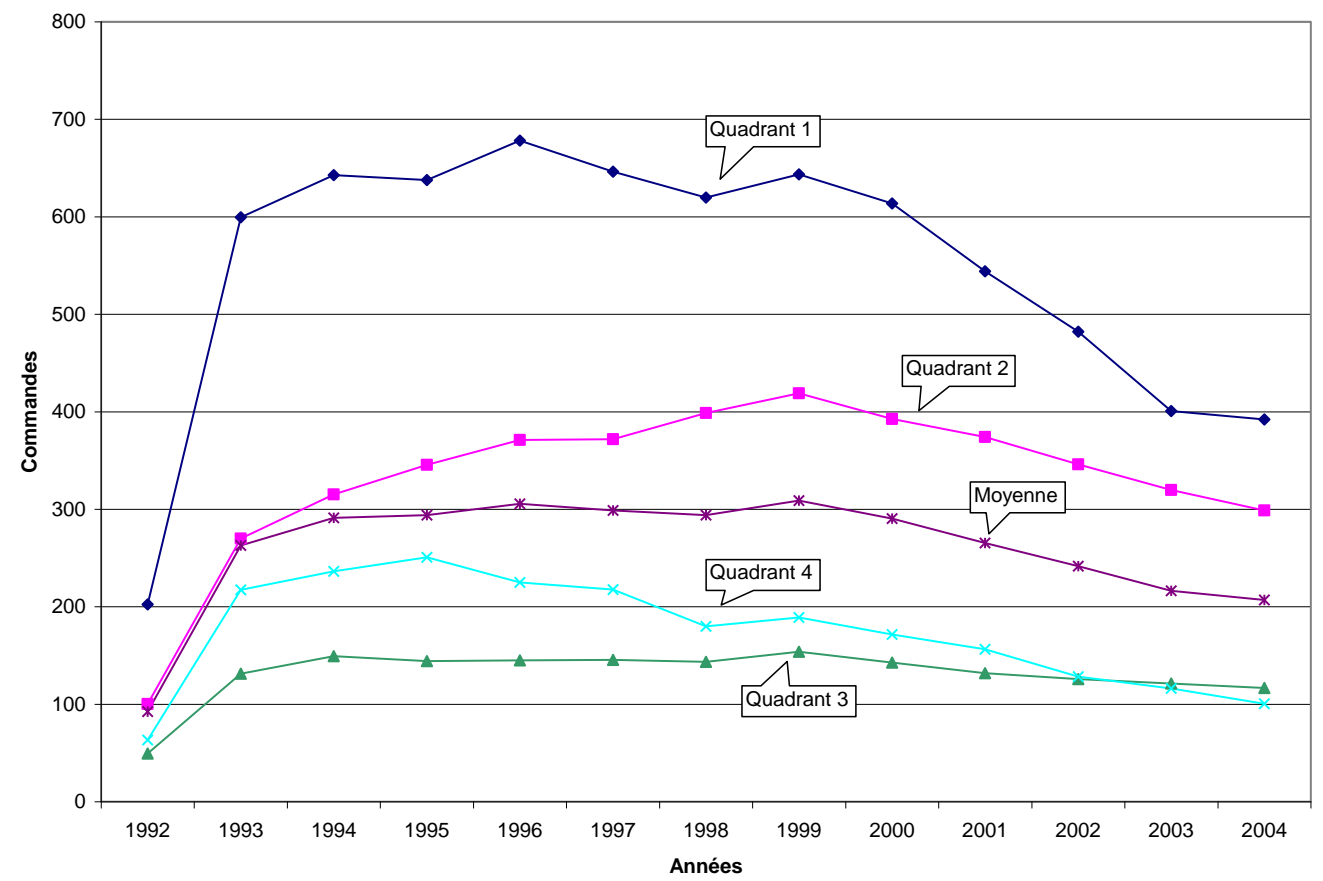

Figure 3. Evolution des commandes des quatre quadrants de 1992 à 2004. 


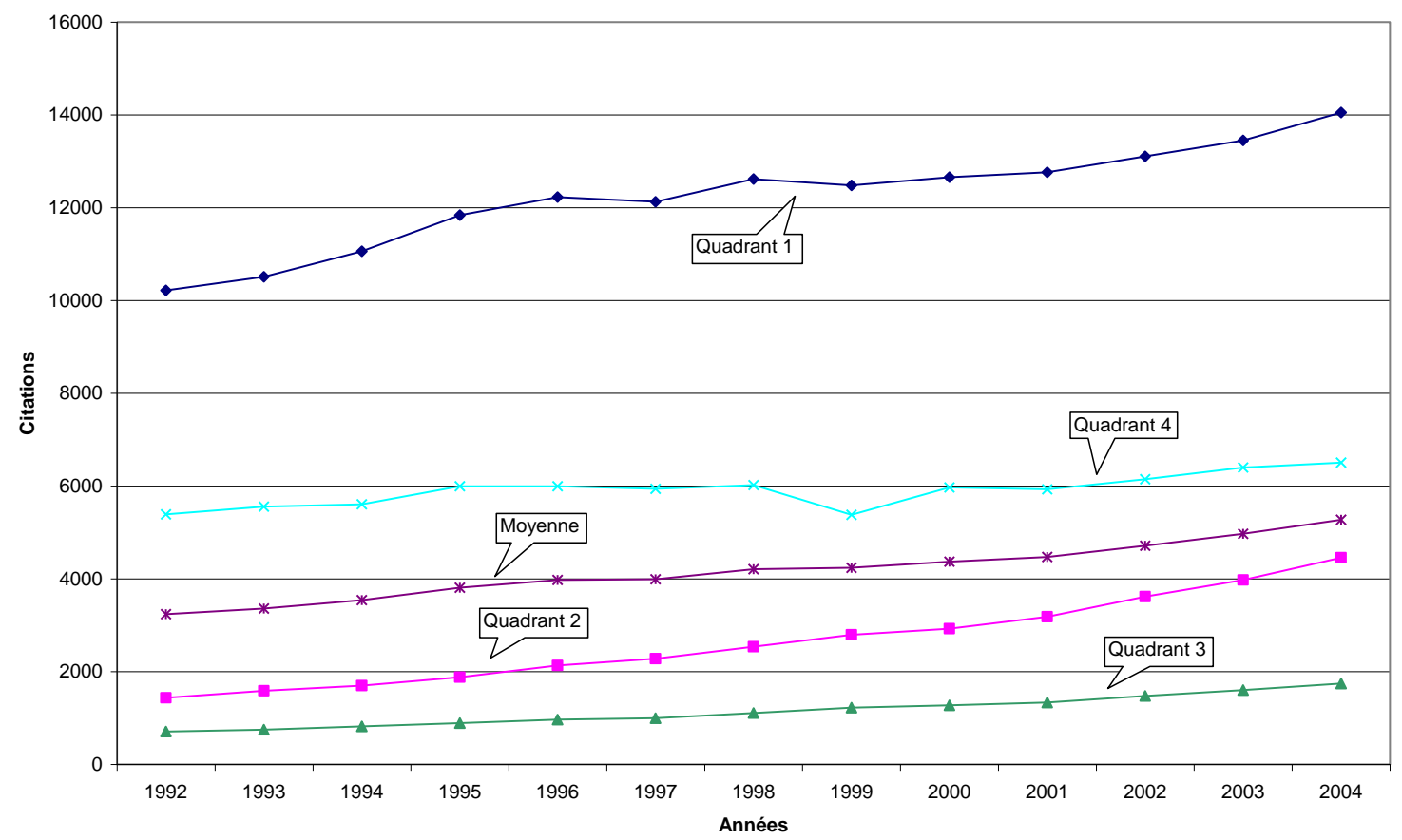

Figure 4. Evolution des citations des quatre quadrants de 1992 à 2004. 


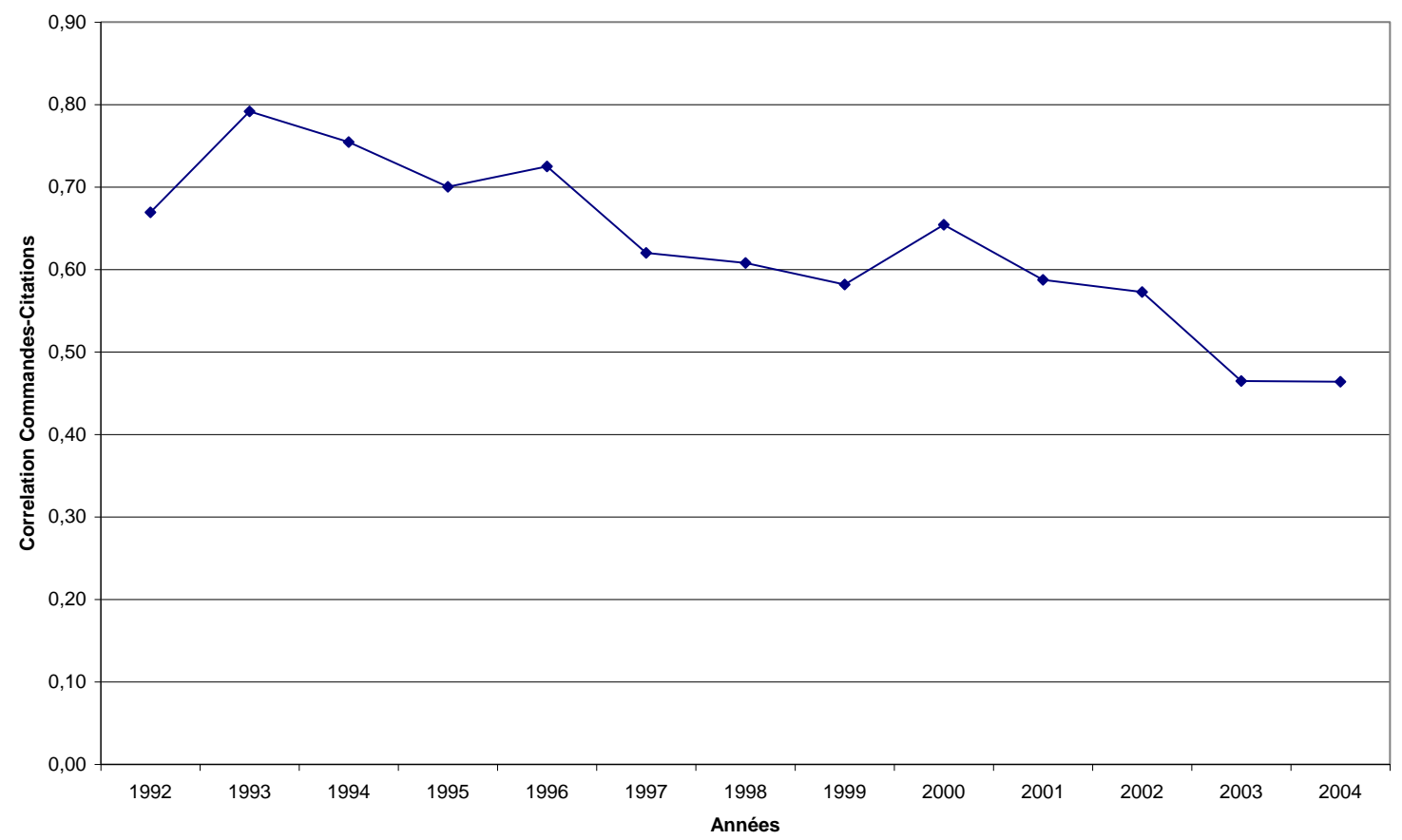

Figure 5. Evolution de la corrélation Commandes-Citations de 1992 à 2004. 